\title{
The genetics of neuroendocrine prostate cancers: a review of current and emerging candidates
}

This article was published in the following Dove Press journal:

The Application of Clinical Genetics

7 November 2012

Number of times this article has been viewed

\section{Hammad Ather' \\ Tahmeena Siddiqui ${ }^{2}$ \\ 'Dept of Surgery, Aga Khan University, ${ }^{2}$ Karachi Medical and Dental College,} Karachi, Pakistan
Correspondence: $\mathrm{M}$ Hammad Ather Aga Khan University, PO Box 3500, Stadium Road, Karachi 74800, Pakistan Tel +92 2l 34864778

Fax +92 2I 34934294

Email hammadather@gmail.com
Abstract: Prostate cancer (PC) displays a strong familial link and genetic factors; genes regulating inflammation may have a pivotal role in the disease. Epigenetic changes control chromosomal integrity, gene functions, and, ultimately, carcinogenesis. The most widely studied epigenetic event in PC is aberrant DNA methylation (hypo- and hypermethylation); besides this, chromatin remodeling and micro RNA (miRNA) are other studied alterations in PC. These all lead to genomic instability and inappropriate gene expression. Causative dysfunction of histone modifying enzymes results in generic and locus-specific changes in chromatin remodeling. miRNA deregulation also contributes to prostate carcinogenesis, including interference with androgen-receptor signaling and apoptosis. These epigenetic alterations have the potential to act as biomarkers for PC for screening and diagnosis as well as prognosis and follow-up. The variable biological potential for a newly diagnosed $\mathrm{PC}$ is one of the biggest challenges. The other major clinical problem is in the management of castration-resistant PC. Neuroendocrine (NE) differentiation is one of the putative explanations for the development of castration-resistant disease. Most advanced and poorly differentiated cancer does not produce prostate-specific antigen (PSA) in response to disease progression. Circulating and tissue biomarkers like chromogranin $\mathrm{A}(\mathrm{CgA})$ thus become important tools. There is the potential to use various genetic and epigenetic alterations and NE differentiation as therapeutic targets in the management of PC. However, we are still some distance from developing clinically effective tools. Valuable insights into the nature of NE differentiation in PC have been gained in the last decades, but additional understanding of its pathogenetic mechanisms is needed. This will help in devising novel therapeutic strategies to develop targeted therapies. $\mathrm{CgA}$ has the potential to become an important marker of advanced castration-resistant PC in cases where prostate-specific antigen can no longer be relied upon. Aberrant androgen-receptor signaling at various levels provides evidence of the importance of this pathway for the development of castration-resistant PC. Many epigenetic influences - in particular, the role of changing miRNA expression - provide valuable insights. Currently, massive sequencing efforts are underway to define important somatic genetic alterations (amplifications, deletions, point mutations, translocations) in PC, and these alterations hold great promise as prognostic markers and for predicting response to therapy.

Keywords: prostate cancer, epigenetic, genetic, neuroendocrine differentiation

\section{Introduction}

It is estimated that there are nearly 2.8 million men living with a history of prostate cancer (PC) in the USA, and that an additional 241,740 cases will be diagnosed in 2012. ${ }^{1}$ The median age at diagnosis is 67 years. ${ }^{2}$ PC affects elderly men and incidence increases with age. The percentage probability of developing PC increases with age: it is $0.01 \%$ for those under 40 years of age, $2.63 \%$ for those aged between 40 and 
59 years, $6.84 \%$ for those between 60 and 69 , and $12.54 \%$ for those aged over 70 years. During 2006-2008, there was a lifetime risk of $16.48 \%$ in the USA. ${ }^{3}$ Similarly, in Europe, PC is the most common solid neoplasm and the most common form of cancer in men $(202,100$ incident cases, $18.1 \%$ of all incident cases in 2004). ${ }^{4}$ One of the remarkable features of $\mathrm{PC}$ is wide variation in incidence across ethnic groups. As an example, there is a high prevalence among African-American (137 cases/100,000 population) compared with the Asian population ( $<10$ cases $/ 100,000$ population) ${ }^{5}$

The mechanisms involved in the development of $\mathrm{PC}$ are still to be determined; however, it is evident that both genetic and epigenetic changes contribute to its development and progression. "Epigenetic" refers to alterations of the DNA or associated proteins, without disturbing the DNA sequence and carrying of information regarding gene expression during cell division. ${ }^{6}$ Altered epigenetic gene regulation is involved in the genesis and progression of PC. Aberrant epigenetic events include DNA hypo- and hypermethylation, chromatin remodeling, altered histone modifications, and changes in micro RNA (miRNA) expression. All these events have been detected in a range of genes associated with PC and produce heritable changes in gene expression without altering the DNA coding sequence. The list of aberrantly epigenetically regulated genes continues to grow; only a few genes have, so far, produced promising results as potential tumor biomarkers for early diagnosis and risk stratification. Epigenetic alterations have the potential to become valuable tools for the management of PC. This is due to reversibility of epigenetic aberrations with modulators that demethylate DNA and inhibit histone deacetylases, leading to reactivation of silenced genes. Pharmacologic compounds that reverse their nature can target these alterations.

In this brief review, effort is made to review the current evidence to improve understanding of the various genetic and epigenetic influences, in particular NE differentiation, in the causation and progress of PC.

\section{Neuroendocrine differentiation in PC}

Neuroendocrine (NE) differentiation in PC represents a heterogeneous group of entities. NE in differentiation has received increasing attention in recent years due to prognostic and therapeutic implications. The NE pattern in castration-resistant prostate cancer (CRPC) may prove useful in selecting potential responders to target therapies such as somatostatin analogues. NE cells are derived from non-NE $\mathrm{PC}$ cells and secrete factors that can act in a paracrine manner to stimulate the survival, growth, motility, and metastatic potential of prostatic carcinoma cells. NE-differentiated PC either involves focal NE cells in the milieu of adenocarcinoma or tumors composed exclusively of NE cells (the rare and aggressive small cell carcinoma and carcinoid/carcinoid-like tumor). There is lack of consensus as to the independent prognostic value of focal NE differentiation in PC; however, a significant body of current evidence suggests that it has an influence on prognosis related to hormone-resistant tumors. There are reports as to its role in the conversion to a hormoneresistant phenotype.

The various transcription factors responsible in the development of NE characteristics through prostatic adenocarcinoma cells include signal transducer and activator of transcription 3, cAMP response element-binding protein, early growth response protein 1, c-Fos, and nuclear factor kappa B. ${ }^{7}$ The different molecular markers of NE cells include chromogranin A ( $\mathrm{CgA}$ ), neuron-specific enolase, B-cell lymphoma 2, and the androgen receptor. ${ }^{8}$ The NE cells secrete a myriad of neuropeptides, which trigger growth and survival responses in androgen-independent PC cells. These neuropeptides are bombesin, neurotensin, parathyroid hormone-related protein, serotonin, and calcitonin. ${ }^{6} \mathrm{PC}$ cell receptors that play a role in these processes include the gastrin-releasing peptide receptor, neurotensin receptors, and the epidermal growth-factor receptor. Signal-transduction molecules activated by these neuropeptides include Src, focal adhesion kinase, extracellular signal-regulated kinases, and phosphatidylinositol 3-kinases/Akt, with subsequent activation of $\mathrm{E}$ twenty-six-like transcription factor 1, nuclear factor kappa B, and c-Myc transcription factors. A multitude of genes are then expressed by PC cells, which are involved in proliferation, anti-apoptosis, migration, metastasis, and angiogenesis. Targeting of these pathways at multiple levels can be exploited to inhibit the process by which NE cells contribute to the progression of androgen-independent treatment-refractory PC.

\section{Genetic factors in the etiology of PC}

In view of the strong ethnic predisposition, strong family association, and familial clustering, there is a strong likelihood that genetic factors play an important role in the development of PC. In the last several years, genome-wide association studies (GWASs) have identified over 36 germ line variants of genes that have shown significant association with PC susceptibility. The wide variation in the incidence of $\mathrm{PC}$ in different ethnic groups supports the genetic etiology 
of PC. ${ }^{5}$ Aside from age and ethnicity, a positive family history is also a significant risk factor. ${ }^{9}$

Over 40 PC susceptibility loci explaining approximately $25 \%$ of the familial risk have been identified. The majority of GWASs reported to date have been conducted in populations of European, African-American, ${ }^{10}$ and Japanese ancestrys. ${ }^{11}$ An up-to-date catalog of published GWASs is available at the US National Institutes of Health National Human Genome Research Institute website (http://www.genome. gov/gwastudies/).

The genetic factors have been used to assess risk of PC development, risk of advanced disease, and duration of response to hormonal therapies. In diagnosis and screening, there is significant potential for the use of urine measurements of prostate cancer antigen $3 \mathrm{RNA}$ and the transmembrane protease like serine $2 \mathrm{v}$-ets erythroblastosis viruses and E26 oncogene homolog (avian) (TMPRSS2-ERG) gene fusion. Multiple groups ${ }^{12,13}$ have developed gene-expression signatures from primary prostate tumors correlating with poor prognosis, and attempts to improve and standardize these signatures as diagnostic tests have been presented. Massive sequencing efforts are underway to define important somatic genetic alterations (amplifications, deletions, point mutations, translocations) in PC, and these alterations hold great promise as prognostic markers and for predicting response to therapy. ${ }^{12}$

\section{Epigenetic factors influencing carcinogenesis}

"Epigenetic alterations" are heritable changes in gene expression that occur without changes in DNA sequence. ${ }^{14}$ Epigenetic changes control chromosomal integrity, gene functions, and, ultimately, carcinogenesis. The aberrant epigenetic events in PC include DNA hypo- and hypermethylation, chromatin remodeling, altered histone modifications, and changes in miRNA expression. The most studied of these is DNA methylation. However, it is not completely understood what induces these epigenetic alterations in cancer; in addition, their role in prostate tumorigenesis is being evaluated. Identification of the epigenetic modifications involved in the development and progression of PC will aid in identifying novel therapeutic targets and indicators of biological and diagnostic markers.

\section{DNA hypo- and hypermethylation}

DNA methylation plays an important role in regulating gene activity, the DNA repair process, and recombination and replication. DNA methylation involves the addition of a methyl group to the $5^{\prime}$-carbon of cytosine in the phosphodiesterase bond between cytosine and guanine $(\mathrm{CpG})$ dinucleotide sequences; a family of DNA methyltransferases catalyzes this step.

DNA methylation can regulate gene activity via two mechanisms. First, methylation of $\mathrm{CpG}$ dinucleotides within transcription-factor binding sites can inhibit transcriptionfactor binding and, therefore, directly influence gene activity. ${ }^{15}$ Secondly, methylated $\mathrm{CpG}$ dinucleotides act as binding sites for methyl $\mathrm{CpG}$ binding proteins, which are associated with other factors such as histone deacetylases, involved in establishing repressive chromatin structures. ${ }^{16}$ These methylation changes have been implicated in the tumorigenesis of PC; however, these changes are complex. They involve both hypo- and hypermethylation. Aberrant DNA hypermethylation occurs when there is an increase in DNA methylation at unmethylated regions. Hypermethylation at gene promoters can lead to its inactivation. In contrast, "DNA hypomethylation" is the demethylation of normally methylated DNA and can lead to chromosomal instability and activation of proto-oncogenes.

\section{Chromatin remodeling and histone modifications}

Chromatin is composed of a protein core, which is made up of eight histones, around which $147 \mathrm{bp}$ of DNA is wrapped. ${ }^{17}$ Histones not only provide physical support to DNA but also are involved in regulating its transcription, repair, and replication. ${ }^{15,16,18}$ Various posttranslational biochemical modifications such as acetylation, methylation, and phosphorylation can potentially affect the histone tail. ${ }^{15,19}$ This results in the histone code, which involves structural and translational changes. These alterations decrease the affinity of histones for DNA. Consequently, an "open" chromatin conformation results and allows the gene transcription to take place. Contrarily, histone deacetylation is associated with closed, or repressive, chromatin. Two enzymes involved in creating an equilibrium state between acetylation and deacetylation are histone acetyltransferases and histone deacetylases. Similarly, histone methylation may be associated with either transcriptional promotion or repression. The histone methylation is modified by two enzymes - histone methyltransferases and histone demethylases. ${ }^{15,16,18}$ In malignant cells, genome-wide histone modification is also altered in concert with changes in DNA methylation. ${ }^{20}$ Interestingly, the same repressive histone marks were identified in certain genes with tumor-suppressor behavior that are not silenced by DNA methylation. ${ }^{21}$ Accordingly, alterations in the expression of key histone-modulating enzymes (histone deacetylases, histone 
acetyltransferases, histone methyltransferases, and histone demethylases) have been associated with cancer development and progression. ${ }^{13,22,23}$

\section{miRNA}

"Micro RNAs" are endogenous non-coding RNAs that can interfere with protein expression either by inducing the cleavage of specific target messenger RNAs or, in most cases, by inhibiting their translation. ${ }^{24}$ Review of the contemporary literature reveals that though over 48 miRNAs have been implicated in PC, only a few have been shown to be of clinical significance. The most significant clinical impact of miRNA is to provide an alternative mechanism sustaining androgen-independent growth. ${ }^{25}$ It has been shown that miRs-221, $-222,-23 b,-27 b,-15 a,-16-1$, and -203 are differentially expressed in the hormone-sensitive LNCaP cell line and the hormone-resistant LNCaP-abl cell line. ${ }^{26}$ This suggests that these miRNAs may characterize certain subtypes of human CRPC. In a recent work, Sun and colleagues suggested that altered miR-221/-222 and miR-23b/-27b expression may be associated with the CRPC process. ${ }^{27}$ However, it has also been suggested that androgens may play a role in downregulation of miR$221 /-222 .{ }^{23}$ Other miRNAs are also potential modulators of androgen receptor-mediated signaling. Besides altering androgen-receptor signaling, miRNAs are also implicated in attributing immortality to PC cells through the avoidance of apoptosis. This is achieved by overexpression of miR- $21^{28}$ and downregulation miR-34a $\mathrm{a}^{29}$ and miR-34c. ${ }^{30}$

\section{Current markers}

The biological potential of PC is highly variable. The majority of PCs is indolent and is diagnosed in the sixth to eighth decades of life. The majority of elderly males is likely to die of other causes and not from cancer. Therefore, it is often unnecessary to subject them to invasive and potentially morbid treatment options. Currently, PSA is the only surrogate marker for diagnosis, prognosis, and follow-up of patients with PC. It is an excellent marker in the follow-up of PC patients managed medically or surgically. However, there are significant issues with PSA's diagnostic, screening, ${ }^{31}$ and predictive/prognostic value. ${ }^{32}$

\section{Potential new markers}

There is an urgent clinical need for the identification of novel biomarkers in PC. Current development in the field of proteomic techniques holds the promise of providing new tools with which to find novel PC biomarkers for early diagnosis and prognosis.

$\mathrm{CgA}$ is an acidic glycoprotein that is commonly expressed by NE cells and constitutes one of the most profuse components of secretory granules. Its concentration is thought to be elevated in relation to NE differentiation of PC. Most advanced PC becomes anaplastic and does not express PSA in tissue or produce PSA in congruence with the increasing bulk of the cancer. In such a situation, PSA becomes an unreliable marker of disease progression. Thus, $\mathrm{CgA}$ is a useful predictive marker in patients with prostatic cancer who have a lower PSA level.

$\mathrm{CgA}$ and PSA are complementary tumor markers in CRPC. ${ }^{33} \mathrm{CgA}$ may help in predicting the response to docetaxel therapy. Rising $\mathrm{CgA}$ levels during therapy may be associated with poor prognosis whereas $\mathrm{CgA}$ response is likely to be associated with clinical response.

It is known that NE cells in the prostate do not contain androgen receptors and are not regulated by androgens. PSA expression can be stimulated by androgen through androgen receptors, so it is suggested that cases of PC associated with low serum PSA and high serum $\mathrm{CgA}$, which would have more NE cells with less androgen receptors, may show resistance to endocrine therapy and a poor prognosis. Therefore, serum $\mathrm{CgA}$ tends to be elevated in high-grade PC cases. Hence, it can be used to fill any gap left by PSA and, when combined with serum PSA, the serum marker may effectively predict prognosis after endocrine therapy. $\mathrm{CgA}$ expression in $\mathrm{PC}$ biopsies is an independent extrapolative factor of hormone-refractory disease in patients with newly diagnosed PC on early androgen-deprivation therapy.

\section{Genetic markers}

The use of genetic markers has the potential to aid disease screening and to improve prognostic discrimination and prediction of response to treatment. However, most markers have not been prospectively validated for providing useful prognostic or predictive information or improvement upon clinicopathologic parameters already in use. Significant efforts are underway to develop these research findings into clinically useful diagnostic tests in order to improve clinical decision-making.

\section{Epigenetic biomarkers}

Among the promising serum epigenetic markers in PC for cancer detection due to DNA methylation are GSTP1, 
PTGS2, RPRM, TIG1, and one related to miRNA - $m i R-141$. The tissue markers related to DNA methylation include GSTP1, APC, PTGS2, and MDR1. Among the various tissue markers used in the prognosis and prediction of the biological potential of the tumor are APC, CD44, PITX2, and PTGS2; H3K4me, H3K4me2, H3K18Ac, EZH2, and LSD1 (DNA methylation); and miRs-34c, -96, -135b, and -194 (miRNA). ${ }^{34}$

\section{Conclusion}

In the last few decades, valuable insights have been gained regarding the nature of $\mathrm{NE}$ differentiation in $\mathrm{PC}$ in the last decades; further understanding of its pathogenetic mechanisms is still required. Understanding of the development and progression of PC, and the factors that drive the development of androgen independence, are better understood in light of NE differentiation. The fact that most cancers eventually produce androgen-independent clones highlights the variety of genetic changes in the primary tumor that result in phenotypically distinct cells with different cellular capabilities, and their own characteristic response to the dynamic microenvironment. This will help in devising novel therapeutic strategies to develop targeted therapies. $\mathrm{CgA}$ has the potential to become an important marker of advanced CRPC when PSA can no longer be relied upon. Aberrant androgen-receptor signaling at various levels provides evidence of the importance of this pathway for the development of CRPC. Many epigenetic influences - in particular, the role of changing miRNA expression - provide valuable insights. Massive sequencing efforts are underway to define important somatic genetic alterations (amplifications, deletions, point mutations, translocations) in PC, and these alterations hold great promise as prognostic markers and for predicting response to therapy.

\section{Disclosure}

The authors report no conflicts of interest in this work.

\section{References}

1. Siegel R, DeSantis C, Virgo K, et al. Cancer treatment and survivorship statistics, 2012. CA Cancer J Clin. 2012;62(4):220-241.

2. Howlader N, Noone AM, Krapcho M, et al, editors. SEER Cancer Statistics Review, 1975-2008. Bethesda, MD: National Cancer Institute, 2011. Available from: http://seer.cancer.gov/csr/1975_2008/. Accessed October 8, 2012.

3. Siegel R, Naishadham D, Jemal A. Cancer statistics, 2012. CA Cancer J Clin. 2012;62(1):10-29.

4. Boyle P, Ferlay J. Cancer incidence and mortality in Europe, 2004. Ann Oncol. 2005;16(3):481-488.

5. Hsing AW, Devesa SS. Trends and patterns of prostate cancer: what do they suggest? Epidemiol Rev. 2001;23(1):3-13.
6. Feinberg AP, Tycko B. The history of cancer epigenetics. Nat Rev Cancer. 2004;4(2):143-153.

7. Amorino GP, Parsons SJ. Neuroendocrine cells in prostate cancer. Crit Rev Eukaryot Gene Expr. 2004;14(4):287-300.

8. Shariff AH, Ather MH. Neuroendocrine differentiation in prostate cancer. Urology. 2006;68(1):2-8.

9. Park JH, Gail MH, Greene MH, Chatterjee N. Potential usefulness of single nucleotide polymorphisms to identify persons at high cancer risk: an evaluation of seven common cancers. J Clin Oncol. 2012;30(17): 2157-2162.

10. Chang BL, Spangler E, Gallagher S, et al. Validation of genome-wide prostate cancer associations in men of African descent. Cancer Epidemiol Biomarkers Prev. 2011;20(1):23-32.

11. Takata R, Akamatsu S, Kubo M, et al. Genome-wide association study identifies five new susceptibility loci for prostate cancer in the Japanese population. Nat Genet. 2010;42(9):751-754.

12. Choudhury AD, Eeles R, Freedland SJ, et al. The role of genetic markers in the management of prostate cancer. Eur Urol. 2012;62(4): 577-587.

13. Fendler A, Jung M, Stephan C, et al. miRNAs can predict prostate cancer biochemical relapse and are involved in tumor progression. Int J Oncol. 2011 Nov;39(5):1183-1192.

14. Berger SL, Kouzarides T, Shiekhattar R, Shilatifard A. An operational definition of epigenetics. Genes Dev. 2009;23(7):781-783.

15. Hark AT, Schoenherr CJ, Katz DJ, Ingram RS, Levorse JM, Tilghman SM. CTCF mediates methylation-sensitive enhancer-blocking activity at the H19/Igf2 locus. Nature. 2000;405(6785):486-486.

16. Jones PL, Veenstra GJ, Wade PA, et al. Methylated DNA and MeCP2 recruit histone deacetylase to repress transcription. Nat Genet. 1998; 19(2):187-191.

17. Jenuwein T, Allis CD. Translating the histone code. Science. 2001; 293(5532):1074-1080.

18. Lennartsson A, Ekwall K. Histone modification patterns and epigenetic codes. Biochim Biophys Acta. 2009;1790(9):863-868.

19. Mellor J. The dynamics of chromatin remodeling at promoters. Mol Cell. 2005;19(2):147-157.

20. Coolen MW, Stirzaker C, Song JZ, et al. Consolidation of the cancer genome into domains of repressive chromatin by long-range epigenetic silencing (LRES) reduces transcriptional plasticity. Nat Cell Biol. 2010; 12(3):235-246.

21. Fraga MF, Ballestar E, Villar-Garea A, et al. Loss of acetylation at Lys 16 and trimethylation at Lys 20 of histone $\mathrm{H} 4$ is a common hallmark of human cancer. Nat Genet. 2005;37(4):391-400.

22. Sharma S, Kelly TK, Jones PA. Epigenetics in cancer. Carcinogenesis. 2010;31(1):27-36.

23. Esteller M. Epigenetics in cancer. New Eng J Med. 2008;358(11): $1148-1159$.

24. Bartel DP. MicroRNAs: genomics, biogenesis, mechanism, and function. Cell. 2004;116(2):281-297.

25. Coppola V, De Maria R, Bonci D. MicroRNAs and prostate cancer. Endocr Relat Cancer. 2010;17(1):F1-F17.

26. Sun T, Wang Q, Balk S, Brown M, Lee GS, Kantoff P. The role of microRNA-221 and microRNA-222 in androgen-independent prostate cancer cell lines. Cancer Res. 2009;69(8):3356-3363.

27. Sun T, Yang M, Chen $\mathrm{S}$, et al. The altered expression of MiR221/-222 and MiR-23b/-27b is associated with the development of human castration resistant prostate cancer. Prostate. 2012;72(10): 1093-1103.

28. Yang CH, Yue J, Fan M, Pfeffer LM. IFN induces miR-21 through a signal transducer and activator of transcription 3-dependent pathway as a suppressive negative feedback on IFN-induced apoptosis. Cancer Res. 2010;70(20):8108-8116.

29. Kojima K, Fujita Y, Nozawa Y, Deguchi T, Ito M. MiR-34a attenuates paclitaxel-resistance of hormone-refractory prostate cancer PC3 cells through direct and indirect mechanisms. Prostate. 2010;70(14): 1501-1512. 
30. Hagman Z, Larne O, Edsjö A, et al. miR-34c is downregulated in prostate cancer and exerts tumor suppressive functions. Int J Cancer. 2010;127(12):768-2776.

31. Wever EM, Hugosson J, Heijnsdijk EA, Bangma CH, Draisma G, de Koning HJ. To be screened or not to be screened? Modeling the consequences of PSA screening for the individual. Br J Cancer. 2012;107(5): $778-784$.

32. Hattangadi JA, Chen MH, D'Amico AV. Early detection of high-grade prostate cancer using digital rectal examination (DRE) in men with a prostate-specific antigen level of $<2.5 \mathrm{ng} / \mathrm{mL}$ and the risk of death. BJU Int. Epub July 3, 2012.
33. Sarkar D, Singh SK, Mandal AK, et al. Plasma chromogranin A: clinical implications in patients with castrate resistant prostate cancer receiving docetaxel chemotherapy. Cancer Biomark. 2010-2011;8(2): 81-87.

34. Jerónimo C, Bastian PJ, Bjartell A, et al. Epigenetics in prostate cancer: biologic and clinical relevance. Eur Urol. 2011;60(4):753-766.

The Application of Clinical Genetics

Dovepress

\section{Publish your work in this journal}

The Application of Clinical Genetics is an international, peer-reviewed open access journal that welcomes laboratory and clinical findings in the field of human genetics. Specific topics include: Population genetics; Functional genetics; Natural history of genetic disease; Management of genetic disease; Mechanisms of genetic disease; Counseling and ethical

issues; Animal models; Pharmacogenetics; Prenatal diagnosis; Dysmorphology. The manuscript management system is completely online and includes a very quick and fair peer-review system, which is all easy to use. Visit http://www.dovepress.com/testimonials.php to read real quotes from published authors. 